\title{
Quand le sang parle en code : origines cachées et bibliques de L'Île aux trente cercueils de Maurice Leblanc
}

\author{
Rebecca Josephy \\ University of Western Ontario
}

Le roman policier a été longtemps accusé d'être une souslittérature, un passe-temps frivole, une entreprise, en somme, anecdotique. Dans ses articles polémiques parus dans le New Yorker d'octobre 1944 et de juillet 1945, «Pourquoi les gens lisent-ils des romans policiers?» et «Que nous importe le meurtre de Roger Ackroyd », Edmund Wilson a soutenu que le roman policier n'était pas fait pour être lu, mais plutôt pour être « parcouru à toute vitesse pour voir comment se résout le problème » (p. 86). Trois ans plus tard, W.H Auden a comparé 
cette littérature à une véritable dépendance: «Pour moi comme pour beaucoup d'autres, la lecture de romans policiers est une drogue, comme le tabac ou l'alcool. » (p.113) Jugés comme un produit de consommation de masse, une littérature à la fois industrielle et industrialisée, les romans policiers notamment ceux précédant les romans réalistes de la "série noire » dont le style hard-boiled de Raymond Chandler et Dashiell Hammett était la préfiguration - ont souffert d'une réputation peu flatteuse.

L'Île aux trente cercueils de Maurice Leblanc occupe une place de premier plan, si l'on peut dire, parmi les œuvres malmenées et mal reçues. Parue tout d'abord en 1919 entre le 6 juin et le 3 août dans le quotidien Le Journal, cette série a été publiée en volume chez Lafitte la même année et a été adaptée en une minisérie télévisée en 1979, diffusée par Antenne 2. Tantôt roman à suspense, tantôt roman fantastique qui glisse dans le Grand-Guignol avec, comme l'indique Francine Marill Albarès, « une inflexion vers le roman d'épouvante » (p. 127), cette œuvre est exemplaire du mauvais goût du roman policier. L'œuvre commence ainsi: en regardant le film Une légende bretonne, l'héroïne, Véronique d'Hergemont, est surprise de voir une cabane abandonnée en Bretagne avec, sur la porte, ses initiales, « $\mathrm{V}$ d'H », écrites exactement à sa façon avec un $d$ minuscule et «la barre de la lettre $H$ ramenée sous les trois lettres » (p.12). Elle décide de creuser plus profond et localise la cabane, qui porte bien ses initiales accompagnées d'un chiffre et d'une flèche. En entrant à l'intérieur, elle trouve une feuille avec un dessin morbide : le croquis, à l'encre rouge, la montre pendue à un arbre à côté de trois autres femmes avec la fatidique inscription : "Trente cercueils et quatre femmes en croix » (p. 12). Elle se met donc à suivre les flèches jusqu'à l'île 
bretonne de Sarek, surnommée par ses habitants «l'île aux trente cercueils ». Une fois là-bas, un secret sur son passé se dénoue : l'enfant auquel elle a donné naissance à dix-sept ans, fruit de ses relations avec un aristocrate cruel, le comte Vorski, et qu'elle a cru mort dans un accident de bateau lorsque son père s'était échappé avec lui, s'avère toujours vivant. Dans l'adaptation télévisée, la musique souligne à grands effets la sentimentalité pathétique de la découverte. Le lecteur apprend, par ailleurs, que le comte a eu un deuxième fils peu de temps auparavant et que ces deux adolescents se ressemblent remarquablement. Dans les chapitres qui suivent, Vorski se met donc à torturer psychologiquement et physiquement son exfemme en utilisant la ressemblance des fils. Il l'enferme dans une prison souterraine d'où elle sort pour assister à un spectacle horrible : celui du duel à mort des deux adolescents, dont elle ignore lequel des deux est son fils. Avant d'avoir rencontré le sosie, Véronique aurait été soumise à une scène grandiose où celui qu'elle croit être son fils lance une flèche en feu sur les habitants de l'île, tuant la majorité des habitants. Il y aura donc pas moins de vingt-six cadavres avant le cinquième chapitre.

Si la présence d'un comte cruel, d'un bain de sang, d'une salle de sacrifice souterraine avec des touches d'anciennes croyances celtiques et bretonnes et des scènes clichées et rebattues du double, l'un diabolique, l'autre angélique - deux frères, deux intrigues romanesques, deux femmes - n'était pas suffisante pour faire basculer ce roman du côté du ridicule et du quasi-pastiche, les derniers chapitres retrouvent le ton du roman policier classique à la Conan Doyle. Le fameux gentleman-cambrioleur, Arsène Lupin, devenu ici détective sous le pseudonyme de Luis Perenna, anagramme de son nom, 
fait son entrée en scène, en authentique deus ex machina du feuilleton. Décrit comme quelqu'un en qui il faut avoir « une confiance absolue, et la certitude qu'en cas de danger l'intervention miraculeuse se produirait à la minute même où elle serait nécessaire» (p.124), l'apparition de ce dernier dissipe tout sentiment de peur chez le lecteur. En moins de deux chapitres, la légende bretonne concernant la croyance en une «Pierre-Dieu », le mystère des quatre femmes en croix et les origines du comte Vorski sont entièrement résolus: Véronique, son fils François et son petit chien « Tout-Va-Bien » finissent par triompher. L'œuvre se clôt avec une phrase des plus niaises: «Nul mieux que toi, délicieux Tout-Va-Bien, ne serait capable de nous montrer, par mille preuves plus convaincantes les unes que les autres, que dans la vie tout s'arrange et que tout va bien... » (p. 284)

Boileau-Narcejac, eux-mêmes écrivains de romans policiers, réserveront ainsi une critique particulièrement virulente à ce roman qui exemplifie, selon eux, la tendance outrancière de certaines œuvres du genre :

Il se produit ici une sorte de phénomène de gigantisme. De même que certains animaux, au cours de l'évolution, ont disparu par suite du développement excessif des dents, des cornes ou de telle autre partie du corps, de même plus d'auteurs qu'on ne croit sont devenus difformes à cause d'un trait d'invention, plaisant au début, puis rapidement hypertrophié. Rex Stout, par exemple, a imaginé Nero Wolfe, ce policier pachydermique qui ne sort jamais de sa maison et ne s'intéresse qu'à ses orchidées. Au bout de quelques romans, il lasse le lecteur. Ellery Queen, lui aussi, manque de mesure et se laisse aller à de telles débauches d'imagination qu'on n'en peut mais. Stanley Gardner tombe dans l'argutie. Chesterton, malgré tous ses dons, finit par s'installer dans l'invraisemblance. Et Maurice Leblanc! Parti de ces ravissantes nouvelles qui sont 
dans toutes les mémoires, il aboutit à ce récit confus étouffant qui s'appelle : L'Île aux trente cercueils. (p. 61)

En toute apparence, ce roman de Leblanc serait donc un cas exemplaire de ces romans de gare dont la critique littéraire n'aurait que faire. Toutefois, si nous évoquons tout ce qui est grand-guignolesque, ridicule et excessif dans cette œuvre, c'est bien parce que cela ne résume pas le roman. Nous verrons, en effet, qu'il s'agit ici d'une réécriture du cinquième chapitre du Livre de Daniel et que ce recours à l'épisode biblique est un phénomène récurrent et peu connu dans l'œuvre de Leblanc, constituant un véritable programme d'écriture. Dans le motif de l'inscription cryptique et le croquis en rouge, sanglant, on découvrira, en effet, une énigme biblique.

\section{"Le Festin de Balthazar»}

Si L'Île aux trente cercueils oscille abruptement et caricaturalement entre différents genres littéraires, le mystère des initiales $\mathrm{V}$ d'H retrouvées sur la vieille porte de la cabane et les fragments d'écriture sur le dessin des quatre femmes en croix sert de fil conducteur au roman. Tout le mystère et le suspense du récit vont reposer, comme l'indique François Bussière, sur le fait que «le père de Véronique s'est abstenu, dans la légende du dessin qui nous est le premier présenté, de reproduire l'intégralité du texte original qu'il a pourtant eu entre les mains; et qui ne sera donné au lecteur, qu'à la fin. La "légende" reste donc bien, jusqu'au bout, ce qui est à lire» (p. 63). Ce motif de l'inscription cryptique, souvent en sang ou en rouge, est présent dans un des présumés ancêtres du roman policier, «Le scarabée d'or » (1843), et trouve ses assises, dans le cas de l'île 
aux trente cercueils, dans l'un des épisodes les plus mystérieux et saisissants de la Bible hébraïque. L'épisode, mieux connu sous le titre "Le Festin de Balthazar», s'ouvre sur une apparition étonnante. Au cours d'un repas organisé par le roi Balthazar, une main détachée du corps humain se met à écrire un message sur le mur du palais :

Soudain apparurent des doigts de main humaine qui se mirent à écrire, derrière le lampadaire, sur le plâtre du mur du palais royal, et le roi vit la paume de la main qui écrivait. Alors le roi changea de couleur, ses pensées se troublèrent, les jointures de ses hanches se relâchèrent et ses genoux se mirent à s'entrechoquer. (Dan 5, 5-6) ${ }^{1}$

Le message laissé par la main qui écrit s'avère d'abord indéchiffrable, poussant le roi à trouver quelqu'un qui soit capable de proposer une interprétation fiable. Après une consultation infructueuse auprès de ses «devins, chaldéens et exorcistes » babyloniens, il recourt à l'un des sages juifs de sa cour, Daniel, qui lit sur le mur les mots suivants : "mené, mené, thecel, parsin » et les participes passés des verbes "mesurer», «peser» et "diviser». Le sens du message devient ainsi : « Mené : Dieu a mesuré ton royaume et l'a livré; Thecel : tu as été pesé dans la balance et ton poids se trouve en défaut; Parsin: ton royaume a été divisé et donné aux Mèdes et aux Perses. » (Dan 5, 26-28) La nuit même, le présage de Daniel s'accomplit lorsque le roi est retrouvé mort et son royaume réparti entre les Perses et les Mèdes.

Le sort de Balthazar peut paraître cruel, mais il s'inscrit tout à fait dans l'esprit des codes et des lois de l'Ancien Testament. Le message sur le mur vient peu de temps après que

${ }^{1}$ La Bible de Jérusalem. 
le roi a demandé de se faire apporter des vases "d'or et d'argent » (Dan 5,3$)$ que son père Nabuchodonosor avait volés du temple hébreu, un événement qui coïncide avec la destruction du premier temple en 586 ans av. J.-C. À l'occasion du festin, Balthazar boit de ces vases sacrés et loue les dieux «d'or, d'argent, d'airain, de bois et de fer» (Dan 5, 4). Les adjectifs «d'or et d'argent » deviennent donc la charnière entre deux morales antithétiques: dans le temple hébreu, les vases ont pour fonction d'honorer un Dieu unique; dans les mains de Balthazar, ils deviennent eux-mêmes des dieux potentiels. Lorsque Daniel interprète l'inscription sur le mur pour Balthazar, il insiste donc sur sa valeur punitive non seulement pour Balthazar, mais aussi pour la dynastie :

Ô roi, le Dieu Très-Haut a donné royaume, grandeur, majesté et gloire à Nabuchodonosor ton père. [...] Mais son cœur s'étant élevé et son esprit durci jusqu'à l'arrogance, il fut rejeté du trône de sa royauté et la gloire lui fut ôtée. [...] Mais toi, Balthazar, son fils, tu n'as pas humilié ton cœur, bien que tu aies su tout cela : tu t'es exalté contre le Seigneur du Ciel, tu t'es fait apporter les vases de son Temple, et toi tes seigneurs, tes concubines et tes chanteuses, vous y avez bu du vin, et avez fait louange aux dieux [...] qui ne voient, n'entendent, ni ne comprennent, et tu n'as pas glorifié le Dieu qui tient ton souffle entre ses mains et de qui relèvent toutes tes voies. Il a donc envoyé cette main qui, toute seule, a tracé cette écriture. (Dan 5, 18-24)

Non seulement s'agit-il alors d'un crime sur la propriété (un vol), mais aussi et surtout, dans le contexte de l'exil babylonien, d'un crime de guerre, dont le but revient à imposer un nouvel ordre, une nouvelle religion, une nouvelle culture. 


\section{Cryptogrammes en formule de trois}

La première indication que Leblanc s'inspire de ce chapitre biblique pour les inscriptions sur le mur dans L'Île aux trente cercueils réside d'abord dans la forme que prend l'écriture. Depuis la traduction biblique de Lemaître de Sacy fondée sur la Vulgate, l'énumération en formule de trois, «mané, thécel, pharès », est devenue l'orthographe la plus standard dans la langue française. L'auteur insiste à plusieurs reprises sur le fait que les initiales de Véronique sont bien composées de trois lettres: "Sur les planches goudronnées de la vieille porte, il y avait tracées à la main, ces trois ${ }^{2}$ lettres: V. d'H. et ces trois lettres, c'était purement et simplement [sa] signature de jeune fille » (p. 12). Le dessin montrant l'héroïne pendue à l'arbre portait à l'extrémité du poteau, et selon la coutume antique, une cartouche avec « une inscription fortement appuyée. Et c'était le paraphe et les trois lettres de Véronique jeune fille, $\mathrm{V}$ d'H : Véronique d'Hergemont!» (p.16) Enfin, des quinze lignes accompagnant le dessin morbide, cette dernière ne peut lire que trois segments: "Quatre femmes en croix"; plus loin: "Trente cercueils..." et, pour finir, toute la dernière ligne ainsi rédigée : "La Pierre-Dieu qui donne vie ou mort". »

L'épisode biblique apparaît ensuite dans le roman par le biais d'une série d'images clés. En entrant dans la cabane, Véronique découvre un cadavre ayant une anomalie particulière: "une des mains de l'homme mort manquait » (p. 15). La main autonome du « Festin de Balthazar » réapparaît alors sous un nouveau jour, présente ironiquement par son absence. Le cadavre est celui, par ailleurs, du villageois

\footnotetext{
${ }^{2}$ Sauf indication contraire, c'est moi qui souligne.
} 
Maguennoc, qui a perdu sa main en touchant une pierre que les habitants croient protégée par les anciens druides de l'île. Cette pierre ressemble curieusement aux vases d'or et d'argent de l'épisode du «Festin de Balthazar» - vases qui, rappelons-le, ont été apportés à Babylone lors de l'exil ${ }^{3}:$ « On dit que la porte, c'est une pierre... et qu'elle vient de très loin, d'un pays étranger... c'est la Pierre-Dieu. On dit aussi que c'est une pierre précieuse qui est d'or et d'argent mélangés. » (p. 60) Elle finit, en outre, par être l'objet d'une tentative de vol par le cruel comte Vorski, parfait miroir, nous allons y revenir, du roi babylonien. Dans une série d'anomalies textuelles ${ }^{4}$ et de références masquées - la répétition du chiffre trois, la composition de la pierre sacrée en « or » et en " argent » et venue de «très loin », l'absence-présence de la main autonome ainsi que des motifs de l'inscription indéchiffrable et du vol - se tissent les fils de l'intertexte biblique.

L'écriture cryptique et le croquis en rouge annoncent, par ailleurs, la mort de Véronique, se dressant comme un présage puissant tel que celui prononcé contre Balthazar. L'œuvre a pour cadre un lieu empreint de mysticisme et dont l'histoire remonterait aux temps anciens. Selon la légende perpétuée de génération en génération, l'île de Sarek vient du nom « sarcophage » et a reçu son surnom de « trente cercueils » d'un lapsus entre « cercueil » et écueils - ces derniers entourant l'île et émergeant comme des masses puissantes «d'un granit noir teinté de rouge, et comme trempé dans du sang» (p.40).

\footnotetext{
${ }^{3}$ « Ayant goûté le vin, Balthazar ordonna d'apporter les vases d'or et d'argent que son père Nabuchodonosor avait pris au sanctuaire de Jérusalem, pour $y$ faire boire le roi, ses seigneurs, ses concubines et ses chanteuses. » (Dan 5, 2) ${ }^{4}$ On pourrait utiliser ici le concept « d'agrammaticalité » ou de «traces » de Riffaterre (1979, p. 29-33, et 1980, p. 4-18).
} 
Chacun des trente écueils correspond à trente tombes sur l'île que les habitants croient réservées pour eux: «les trente cercueils auraient leurs trente victimes, mortes de mort violente...» (p. 117) À cette légende des trente cercueils s'ajoute celle de la Pierre-Dieu. Le présage veut que les druides reviennent pendant une nuit sanglante afin de la protéger. Maguennoc, décrit comme une sorte de prophète «à la fois sorcier et rebouteux, guérisseur et charlatan » (p.117), prédit qu'il sera le premier à périr, suivi par le père de Véronique et par les autres habitants de l'île et les quatre femmes en croix. La même inscription que Véronique retrouve sous le dessin morbide la montrant pendue à un arbre apparaît, en effet, également sur l'une des tombes, surnommée le Dolmen-auxFées: "Trente cercueils et quatre femmes en croix. La PierreDieu qui donne vie ou mort » (p. 17). Avec chaque nouveau mort, suivant l'ordre annoncé par le présage fatidique tel que l'interprète Maguennoc, l'héroïne se croit vouée à un destin inéluctable: «Tout se passait selon la logique implacable de faits [...], liés les uns aux autres comme les mailles d'une chaîne. » (p. 83) Lorsqu'elle découvre un arbre dépouillé de ses branches inférieures et sur lequel trois femmes sont clouées les bras écartelés comme des crucifiées (p. 85-86), tout semble s'avancer implacablement vers un futur déjà écrit et qui renvoie, de plus, à un troisième présage prononcé lors de son mariage avec Vorski. Ce dernier ne cessa de répéter, telle l'inscription sur le Dolmen-aux-Fées : «Ma femme mourra sur la croix. »

Ce double topos du présage fatidique et de personnages remplissant un destin dorénavant écrit ne se limite pas à L'île aux trente cercueils. "Ces horloges fatales », telles que les nomme François Raymond, «vont scander l'ensemble des 
"Aventures extraordinaires d'Arsène Lupin", et être associées de plus en plus étroitement aux "pré-dictions" qui en constituent si souvent le pivot, condamnant le présent à répéter le passé» (p. 42) . Dans Le Triangle d'or (1919), "Patrice et Coralie » peuvent d'avance « lire le déroulement de leur propre agonie dans l'inscription que leur [ont] léguée les précédents "Patrice et Coralie"» (ibid., p.43). Dans Les Dents du tigre (1920), par exemple, le premier indice s'avère être une inscription de trois lettres laissée sur le bureau du préfet de police et qui forment, selon l'hypothèse du détective, le nom de famille des prochaines victimes : FAU. Dans Dorothée danseuse de corde (1923), le père de Dorothée répète fatalement le nom « Roborey » à sa mort et les trois premières lettres du nom se trouvent sur la serrure d'un coffre de trésor, « R.O.B ». Dans ces romans, comme d'ailleurs dans bien d'autres, il s'agit donc d'un message prophétique qui, comme l'écriture sur le mur babylonien, annonce la mort.

Si ces divers romans évoquent les thèmes et motifs de Daniel 5, c'est avec un roman picaresque moins connu, intitulé La Vie extravagante de Balthazar (1925), que l'obsession de Leblanc pour des cryptogrammes fatidiques et en formule de trois se rattache le plus explicitement à Daniel 5. L'intrigue a pour protagoniste un philosophe nommé Balthazar ${ }^{6}$ portant les initiales MTP tatouées sur sa poitrine - un acronyme pour "Mané Thecel Pharès »; c'est avec le concours de l'agence

\footnotetext{
${ }^{5}$ Si Raymond ne mentionne jamais l'épisode du « Festin de Balthazar », sa fine analyse de la temporalité dans l'œuvre de Leblanc ainsi que son emploi d'italiques pour les mots «inscription » et «pré-dictions » renvoient aux principaux enjeux de ce chapitre biblique de Daniel.

${ }^{6}$ Contrairement à Bordillon (1976), qui voit dans Balthazar un des rois mages, nous pensons qu'il s'agit bien ici du roi babylonien du Livre de Daniel.
} 
«XYZ » qu'il découvrira la signification de ces lettres. Ce roman, dont les cryptogrammes se multiplient et foisonnent de possibilités, s'annonce, de fait, dans l'exergue comme un pastiche du roman policier :

La ligne est vague et conventionnelle entre ce qui est vraisemblable et ce qui ne l'est point. Il suffit de bien peu de chose pour qu'une œuvre d'imagination tourne vers la parodie et que des personnages qu'on a voulu pathétiques fassent figure comique et absurde. (p. 6)

«Le Festin de Balthazar » s'impose ainsi dans l'œuvre de Leblanc comme un véritable modèle du roman policier: une source d'inspiration pour ses innombrables combinaisons et manipulations de lettres, de mots et de chiffres, mais aussi et surtout pour son recours systématique à une temporalité de l'accomplissement ou de la préfiguration. Gravés littéralement dans le marbre, les personnages sont voués à répéter le passé jusqu'à ce que Lupin ou son substitut entre en scène pour démêler le mystère en lisant et en déchiffrant les signes correctement, comme l'avait fait Daniel7.

${ }^{7}$ Dans la préface de Messac (2011), on trouve la présentation suivante
de Claude Amoz: «Au lieu de s'en tenir à la facilité et à limiter son
étude au XIX siècle, qui voit l'avènement du genre, Régis Messac
sollicite l'antiquité la plus haute. Emporté par sa démonstration, le
lecteur découvrira que Dupin et Sherlock Holmes ont eu un
prédécesseur aussi illustre qu'inattendu, le prophète Daniel. » (p. 14)
Si, dès le plus jeune âge de la critique, Le Livre de Daniel a été associé
au roman policier, les chercheurs de cette période (Messac, Wrong,
Sayers et Haworth) se sont concentrés uniquement sur deux épisodes
deutérocanoniques dans Daniel: «Suzanne et les vieillards » et «Bel
et le serpent ». Mes recherches actuelles cherchent àmontrer que «Le
Festin de Balthazar», avec son motif de l'inscription cryptique et du
code, constitue également un précurseur significatif dans le
développement du genre, influençant directement un nombre 


\section{Des destins stéréotypés ou l'empreinte de la Première Guerre mondiale}

Si le motif de l'inscription cryptique sur le mur est un élément récurrent dans l'œuvre de Leblanc et apparaît au moins depuis 1909 dans L'Aiguille creuse $^{8}$, le recours à l'épisode biblique gagne en importance et devient la marque d'un destin de plus en plus fatidique dans une série de romans publiés après 1914 : Le Triangle d'or, Les Dents du tigre, auquel s'ajoute, bien sûr, L'Île aux trente cercueils. Si le roman est paru en 1919, l'intrigue se déroule en 1917, une donnée importante et soulignée dans l'exergue :

Aventure authentique, cependant, bien que certains détails prennent, au premier abord, une apparence en quelque sorte fabuleuse. Mais la guerre a compliqué l'existence au point que des événements qui se passent en dehors d'elle, comme ceux dont le récit va suivre, empruntent au grand drame quelque chose d'anormal, d'illogique et parfois, de miraculeux. (p. 8)

Figurant donc à l'arrière-plan, la guerre apparaît d'abord en filigrane - le tuteur de François qui boîte, par exemple, ou encore, à travers l'image des nombreux réseaux souterrains, tunnels et chambres, habités par un ennemi caché, qui rappellent à bien des égards cette guerre qui se déploie dans l'obscurité des tranchées. Elle s'instaure ensuite plus explicitement dans le récit lorsque Maguennoc prévoit que les

important d'écrivains de romans policiers tels que Conan Doyle, Leblanc, Christie, Butor et Eco, pour n'en nommer que quelques-uns.

${ }^{8}$ Il est difficile de savoir si Leblanc s'inspire du «Festin de Balthazar » dans cette œuvre, car la dimension fatidique des inscriptions, si caractéristique de l'épisode biblique et d'où provient la célèbre expression anglaise : "The writing is on the wall », n'apparait pas. 
catastrophes de la légende de Sarek auront lieu en 1917 : «Il prétendait [...] que la dernière ligne de l'inscription sur le Dolmen-aux-Fées n'était pas encore effacée et que l'on pouvait lire de ce vers qui rimait avec "les femmes en croix" : "Dans l'île Sarek, en l'an quatorze et trois" » (p. 118). Cette affirmation devenait d'autant plus impressionnante pour Maguennoc que «le nombre total se divisait en deux nombres, et que précisément en 1914 éclata la guerre » (p. 118). Enfin, ce conflit devient le moteur du récit lorsque le lecteur apprend que Vorski a caché sa véritable identité, qu'il est un espion allemand et que son nom de famille est Hohenzollern, le nom même de la famille impériale. Il est le fils de Louis II, le roi fou de Bavière, qui profite et se nourrit des croyances des habitants de l'île afin de torturer son ex-femme et de voler aux alliés la Pierre-Dieu, qui n'est autre qu'une pierre radioactive.

Le roman s'inscrit ainsi dans une vague d'œuvres patriotiques publiées entre 1914 et 1919 et qui ont, comme le note Dominique Kalifa, «intériorisé la guerre et son contexte » (p. 139). Kalifa estime que « $43 \%$ des "rez-de-chaussée" publiés par les "Quatre Grands" [quotidiens] » ont été consacrés à des fins propagandistes (p. 137-138). Dès lors, le détective, généralement une figure individualiste, distincte de la police par son titre d'enquêteur " privé », assume le rôle de défendeur des intérêts nationaux. Les frontières entre roman policier, roman d'espionnage et roman populaire se brouillent, de même que celles entre crimes individuels et crimes de guerre. Vols, meurtres, viols perpétués par un individu vont se mêler aux crimes d'un pays lorsque les exploits militaires de la Triple Alliance sont criminalisés dans la presse et dans la fiction. L'Allemand, comme le soulève François Gugelot en citant un passage de L'Éclat d'obus de Leblanc, est 
invariablement décrit comme une bête sanguinaire, un barbare, négatif du soldat français : "Quoi qu'on dise, c'est un barbare, une brute orgueilleuse, un homme de sang et de proie. [...] Tout ce que je peux te dire ne vaut l'effroyable réalité. Ce sont des monstres. » (p. 20)

Cette figure du monstre va continuer, bien sûr, jusque dans L'Île aux trente cercueils lorsque, sous les ordres de Vorski, vingt-six habitants de l'île sont sacrifiés d'un seul coup. Les habitants qui ont survécu à la menace initiale sont ensuite traqués et décimés comme des animaux lors d'une chasse : « On abat le gibier ...Ah! les pauvres gens de Sarek! » (p. 65) C'est une boucherie hors de toutes proportions qui contribue à promouvoir l'image de l'Allemand comme inhumain, un être sans conscience qui tire du plaisir de ses crimes. "L'essentiel pour [Vorski] », accuse Lupin, "c'est de frapper à tour de bras, de [se] griser à la vue et à l'odeur du sang, et, avec de la chair vivante, de faire une abominable bouillie. » (p. 224)

Le roman devient ainsi un puissant hymne nationaliste, ayant une fonction très semblable à celle de l'épisode biblique, soit celle de réunir le peuple et de dénigrer l'ennemi. « Le Festin de Balthazar », rappelons-le, a lieu dans la cour babylonienne après la destruction du Premier Temple et durant la période de l'exil. Le Livre de Daniel a été compilé, cependant, à l'époque hellénistique (vers 165 ans av. J.-C.) au moment de la persécution séleucide et des programmes d'assimilation d'Antiochos IV, un chef brutal, dont la tradition chrétienne fera ultérieurement une figure d'antéchrist. Au moment de sa compilation au deuxième siècle av. J.-C., Daniel 5 aurait donc été lu à la lumière de cette menace et l'empire babylonien se dresserait dans la conscience du peuple hébreu comme un reflet du pouvoir grec. Chez Leblanc, la menace se manifeste 
sous un nouveau jour: elle prend les traits de l'Allemand. Balthazar, symbole ultime du despote, revit donc dans cette figure du « Superboche » (p. 231) .

Les parallèles entre Vorski et Balthazar sont, en effet, nombreux et explicites. La débauche et l'excès de la scène biblique - les vases sacrés remplis de vin, le roi qui boit copieusement et orgueilleusement devant ses concubines, ses femmes et ses invités - cèdent la place à un Vorski devenu alcoolique: «Le monstre est fou. Il est fou, et il est ivre. Le dénouement approche. Il boit, il boit [...].» (p. 254) Vaniteux, maniaque et se désignant lui-même à la troisième personne, celui-ci incarne pleinement et caricaturalement les traits du roi babylonien et le comportement menant à son châtiment ${ }^{10}$. De

9 La tendance à présenter Balthazar comme l'archétype du tyran est particulièrement prégnante dans la littérature de la fin du XIX ${ }^{\mathrm{e}}$ et du $\mathrm{XX}^{\mathrm{e}}$ siècle. Dans Les Châtiments de Victor Hugo, Balthazar se métamorphose en Napoléon lorsque la date du 18 brumaire s'écrit en majuscule sur le mur. Dans la Trilogie transylvaine de Miklos Banffy (1934-1940), ayant comme titres individuels Vos jours sont comptés, Vous étiez trop léger et Que le vent vous emporte, Balthazar est incarné dans la classe dominante de la Hongrie qui dîne, danse, boit et parie sa richesse. Dans « Le Festin de Balthazar » de Fazil Iskander, le roi babylonien se métamorphose en Staline. Sous la plume de Klaus Mann, Hitler est la figure du despote. Mann intitule le chapitre consacré aux années 1930-1932 de son autobiographie Le Tournant: L'histoire d'une vie «L'inscription sur le mur». Comme l'indique Sylvie Parizet, il n'y a rien d'étonnant «à ce que cette période d'intenses bouleversements ait vu proliférer le recours au mythe à des fins idéologiques. La Grande Guerre, la Révolution russe, l'éclatement de l'Empire austro-hongrois, la naissance de l'empire américain, la montée des totalitarismes, la Shoah, Hiroshima, la décolonisation, le joug communiste... autant de douloureuses remises en question qui incitent les écrivains à faire appel à de grandes figures mythiques "revisitées" » (p. 18).

${ }^{10}$ L'orgueil et l'excès, principaux traits associés à Balthazar, ont été repris dans le poème «Balthazar» d'Heinrich Heine. Dans celui-ci, le roi provoque Dieu pendant que ses troupes de vassaux l'encouragent, buvant allègrement et à l'excès : « Jehovah! Contre toi je proclame un défi éternel - Je suis le roi de Babylone! » 
l'épisode biblique, Leblanc emprunte ainsi la figure du despote, mais aussi l'idée d'un peuple maudit. Vorski est décrit à plusieurs reprises comme «le fils du roi», un renvoi à Balthazar, «fils de Nabuchodonosor». Le déchiffrement de l'énumération "mané, thécel, pharès » repose, rappelons-le, sur la capacité de Daniel à voir le crime à la lumière d'une filiation : Nabuchodonosor qui a détruit le temple, puis Balthazar qui profane les vases sacrés. De père en fils s'instaure ainsi une transmission qui se traduit, chez Leblanc, dans le rapport entre Louis II de Bavière et Vorski. Il ne s'agit pas simplement d'une seule mauvaise graine, mais d'une culture de la destruction, une race qui, dans l'optique de l'auteur, est prédestinée à commettre les crimes les plus odieux.

N'oublions pas non plus que cette guerre témoigne aussi d'une longue montée de tension commençant dès le lendemain de la guerre franco-prussienne. Toute la série des Lupin pourrait, en effet, être lue comme l'évolution d'un vague anarchisme ${ }^{11}$ vers un nationalisme revanchard, aboutissant en dernier lieu à un respectable détective privé. À partir de son roman 813 (1910) ${ }^{12}$, le cycle patriotique débute : Lupin tente de négocier la restitution de l'Alsace et de la Lorraine avec Guillaume II et réussit à obtenir plusieurs concessions coloniales. Dans Le Triangle d'or, le héros, malin, défend les intérêts nationaux lorsqu'Essarès-Bey cherche à vider le pays de 300 millions de francs or. Cette somme est ensuite livrée au gouvernement français, qui l'utilise pour financer l'entrée en

\footnotetext{
${ }^{11}$ Dans les premiers romans de la série, Lupin ressemble à une sorte de Robin des bois français et partage quelques traits avec Alexandre Jacob. Voir notamment Kalifa (1995, p. 220).

12 Le texte a été réédité en 1917 et modifié pour ajouter des diatribes antiallemandes.
} 
guerre de leur alliée, l'Italie. La miraculeuse " Pierre-Dieu », quant à elle, n'en est pas moins vitale pour les intérêts nationaux, anticipant les puissants usages de la radioactivité et de l'atome. De roman en roman, le patriotisme se fait donc de plus en plus exacerbé et prend un tour mystique avec la défense de la Pierre-Dieu, figuration de la défense du sol sacré de la Patrie, chère à la propagande guerrière.

En dépit d'une sécularisation sociétale importante, le conflit s'exprime, comme le montre Annette Becker, dans des termes profondément religieux :

De 1914 à 1918, sans interruption [...], la guerre menée par l'Allemagne, c'est celle des "atrocités" qui, réelles ou supposées, sont bien entendu toujours acceptées comme vérité historique et symboles de l'éthique allemande: infériorité morale, voire démonomanie. Au nom de Dieu et de la France, le militant de la foi se bat contre cette mort négative, cette mort diabolique. (p. 17)

Vorski revendique ce "germanisme » maléfique : «C'est Dieu qui m’a inspiré, le Dieu de Germanie, le vieux Dieu allemand, pour qui le bien et le mal ne comptent pas quand il s'agit de la grandeur de ses fils. L'esprit du mal est en moi. J'aime le mal et je veux le mal. » (p. 162) Un peu plus loin, Lupin se penche sur sa propension à faire couler le sang en le décrivant comme un "prophète rouge» (p. 202) et en se moquant de sa mégalomanie: "C'est l'Ancien et le Nouveau Testament, le Livre Saint, qui explique et qui commente la loi de Sarek! C'est l'Évangile même de la Pierre-Dieu. Et cet Évangile le désigne, lui, le Superboche, comme le Messie chargé d'accomplir les décrets providentiels. » (p. 248) À ce faux prophète s'oppose, cependant, le héros français. Déguisé en druide, Lupin triomphe en utilisant lui aussi des croyances anciennes : « Il y 
avait, l'une en face de l'autre, deux forces miraculeuses, l'une émanant de lui, Vorski, l'autre émanant du vieux Druide, et la seconde absorbait la première.» (p.215) Deux hommes agissant ainsi en vrais "surhommes", l'un - allemand incarnant le Mal, l'autre - français-, le Bien. Ce combat ne se joue donc plus sur le seul plan territorial mais atteint à un niveau véritablement théologique.

Comme bien des critiques l'ont déjà montré, le roman policier témoigne intrinsèquement d'une tendance à rétablir un ordre perturbé, mais chez Maurice Leblanc, l'idée prophétique d'un destin historique prédomine et atteint des niveaux audacieux. À la fois justicier et sauveur, Lupin s'apparente, par sa mission, à un homme providentiel. Comme le note JeanClaude Vareille, il « lit son destin dans les astres et les astres lui parlent. Il prend sa place dans le Grand Tout.» (p.53) Sous l'identité de Don Luis Perenna, "mystérieux comme Monte Cristo » (1986 [1920], p. 13), le héros français accomplit une vengeance nationale, s'emparant du stylo divin et traçant luimême les lettres de la sentence. Du personnage dumassien ${ }^{13}$, Maurice Leblanc reprend, ainsi, non seulement l'idée d'un individu agissant en tant que Dieu, mais aussi celle d'un châtiment implacable qui s'écrit depuis l'origine du crime et dont la sentence n'est révélée qu'à la fin du récit ${ }^{14}$. Le nom «Vorski de Hohenzollern » forme les mêmes initiales fatidiques

\footnotetext{
${ }^{13}$ Pour une excellente analyse de la filiation entre l'œuvre de Dumas et celle de Leblanc, voir Frigerio (2002).

14 Le Comte de Monte-Cristo contient plusieurs références au «Festin de Balthazar » et pourrait lui aussi être vu comme une réécriture de cet épisode biblique puisque les lettres dénonciatrices qui scellent le destin de Dantès et qui déclenchent toute l'intrigue de revanche sont décrites sous cette lumière : « chaque ligne flamboyait sur la muraille comme le Mane, Thecel, Pharès de Balthazar» (p. 143). Voir également le chapitre « La main de Dieu ».
} 
que celles de sa victime : VDH. À la fin, c'est donc, bien entendu, le comte que nous retrouverons pendu à l'arbre, puni pour ses péchés et accomplissant ce dessein divin.

Loin alors d'être simplement la mise en scène d'un bain de sang, un déferlement de violences gratuites, L'île aux trente cercueils cache par son intertextualité biblique l'idée d'une justice divine et d'un accomplissement prophétique qui traverse toute l'œuvre de Leblanc. Sa nature d'œuvre patriotique et propagandiste fonctionne sur le registre de l'excès et des clichés. Il serait donc facile de ne voir dans l'usage de la Bible qu'un trait grossier et une succession de stéréotypes de littérature à deux sous, car il n'en manque pas ici. Si le recours à la Bible était, en effet, la marque d'une bonne œuvre, bien des dime novels se trouveraient sur des listes de chefsd'œuvre de la littérature. Il faudrait, cependant, tenir compte de ce que la présence de l'intertexte biblique révèle de certains des traumatismes profonds de l'époque. Dans le contexte de la Grande Guerre, le sang coulé devient non seulement la preuve d'une boucherie imputée à l'ennemi, considéré comme inhumain, mais aussi un attribut « racial » : c'est à son sang que l'on reconnaît «l'Allemand». À l'instar du récit biblique, le destin des héros devient donc celui des nations dont ils sont les ressortissants. Arsène Lupin doit non seulement vaincre, mais aussi panser les plaies d'une société qui a souffert. La petite communauté de Sarek est décimée, mais victorieuse, comme le sont les Français et la justice s'accomplit dans un simulacre d'exécution symbolique que l'énigme initiale avait déjà inéluctablement annoncé. En effet, la façon dont Véronique écrit ses initiales avec la «barre de la lettre $h$ ramenée sous les trois lettres » n'évoque-t-elle pas la silhouette de la guillotine, de l'échafaud, sans oublier l'homonymie entre la lettre «h » et la 
hache $^{15}$ ? Si les aventures de Lupin ont donc une fonction de divertissement et restent en grande partie de «la littérature de gare », elles retrouvent cependant, à la faveur d'événements historiques majeurs, l'une des fonctions du texte biblique, qui est de populariser et de donner un sens aux questions que se posent les fidèles lorsqu'ils sont confrontés à une tragédie historique. En ce sens, le sang prend ici une dimension supplémentaire - ce n'est pas seulement un accessoire de Grand-Guignol, mais aussi le sceau du temps présent.

\section{Bibliographie}

AlBÉRES, Francine Marill. (1979), Le Dernier des dandies, Arsène Lupin, Paris, A.G. Nizet.

AUDEN, W.H. (1983 [1948]), « Le presbytère coupable », dans Uri Eisenzweig (dir.), Autopsies du roman policier, Paris, Union Générale d'Éditions, p. 113-132.

BECKER, Annette. (1994), La Guerre et la foi: De la mort à la mémoire 1914-1930, Paris, A. Colin.

Boileau-NARCEJAC. (1975), Le Roman policier, Paris, Presses universitaires de France.

BordilloN, Henri. (1976), «Arsène Lupin, Balthazar et Dorothée », Europe, no 604, p. 78-84.

BuSSIÈRE, François. (1976), "Arsène Lupin: homme de lettres

(l'île aux trente cercueils) », Europe, no 571-572, p. 61-71.

Collins, J.J. (1993). Daniel: A Commentary on the Book of Daniel, Minneapolis, Fortress Press.

${ }^{15}$ Cette image est un emprunt évident à Victor Hugo, qui avait aussi l'habitude de jouer de son nom et de ses initiales dans des dessins comme dans certains écrits. 
DÉRouARD, Jacques. (1989), Arsène Lupin malgré lui, Paris, Séguier.

DubourG, Maurice. (1979), "Arsène Lupin, témoin de son temps et de l'histoire », Europe, no 604, p. 12-20.

DumAS, Alexandre. (1981 [1844]), Le Comte de Monte-Cristo, Paris, Gallimard.

Frigerio, Vittorio. (2002), Les Fils de Monte-Cristo, Limoges, Presses universitaires de Limoges.

GugELOT, Frédéric. (2003), "Roman policier, roman d'espionnage : franchir une frontière (1913-1919)", dans Nathalie Martinière et Sophie Le Ménahèze (dir.), Écrire la frontière, Limoges, Presses universitaires de Limoges, p. 1530 .

HEINE, Heinrich. (1999 [1827]), « Balthazar», dans Le Livre des chants, trad. Nicole Taubès, Paris, Cerf.

Kalifa, Dominique. (1998), "Guerre, feuilleton, presse 19131920», 14-18 Aujourd'hui, Today, Heute, no 2, p. 128-141.

-. (1995), L'Encre et le sang: récits de crimes et société à la Belle Époque, Paris, Fayard.

LEBLANC, Maurice. (1986 [1909]), L'Aiguille creuse, dans Arsène Lupin, t. 1, Paris, Robert Laffont.

—. (1986 [1919]), Le Triangle d'or, t. 2, Paris, Robert Laffont.

—. (1969 [1919]), L'île aux trente cercueils, Paris, Livre de poche.

—. (1986 [1920]), Les Dents du tigre, t. 3, Paris, Robert Laffont.

-. (1986 [1923]), Dorothée danseuse de corde, t. 4, Paris, Robert Laffont.

—. (1979 [1925]), La Vie extravagante de Balthazar, Paris, Livre de poche. 
MESSAC, Régis. (2011 [1929]), Le "Detective Novel" et l'influence de la pensée scientifique, Paris, Encrage.

PARIZET, Sylvie. (2007), "Introduction», dans Sylvie Parizet (dir.), Lectures politiques des mythes littéraires au XXe siècle, Paris, Presses universitaires de Paris Ouest, p. 13-23.

PoE, Edgar Allan. (2009 [1843]), Le Scarabée d'or, trad. Charles Baudelaire, Paris, Éditions Hatier.

RAYMOND, François. (1979), "Arsène Lupin et le démon de la répétition », Europe, no 604, p. 42-50.

Riffaterre, Michel. (1979), La Production du texte, Paris, Seuil.

—. (1980), «La trace de l'intertexte », La Pensée, vol. 215, p. 418.

Tourteau, Jean-Jacques. (1970), Arsène Lupin à San-Antonio, Paris, Mame.

VAREILLE, Jean-Claude. (1979), « Modernité et tradition», Europe, no 604 , p. 50-55.

WILSON, Edmund. (1983 [1944]), « Pourquoi les gens lisent-ils les romans policiers? », dans Uri Eisenzweig (dir.), Autopsies du roman policier, Paris, Union Générale d'Éditions, p. 82-89.

—. (1983 [1945]), "Que nous importe le meurtre de Roger Ackroyd », dans Autopsies du roman policier, p. 90-112.

\section{Résumé}

Si la Première Guerre mondiale n'arrête pas toute production littéraire, elle a néanmoins un impact immédiat sur les thèmes abordés et provoque une surenchère propagandiste et nationaliste. Les littératures policières et populaires en général n'échappent pas à ce phénomène. C'est dans ce cadre que nous étudions la réception particulière d'un épisode biblique connu 
sous le titre "Le Festin de Balthazar» dans les œuvres de Maurice Leblanc et, en particulier, dans L'île aux trente cercueils. Cette étude sera donc l'occasion de découvrir que la reprise du chapitre 5 du Livre de Daniel constitue un surprenant et peu connu programme d'écriture dans l'œuvre du créateur d'Arsène Lupin.

\begin{abstract}
If literary production does not entirely cease during the First World War, it nonetheless shifts focus, giving rise to a significant increase in nationalistic and propagandist material. Detective fiction and popular literature in general are not immune to this phenomenon. It is in this light that we study the reception of a biblical episode known colloquially as «Belshazzar's Feast » in the works of Maurice Leblanc, and in particular in L'Île aux trente cercueils. Here we will discover that the fifth chapter of The Book of Daniel constitutes a surprising and little known intertextuality running through some of the most well-known works of the creator of the eminently French Arsène Lupin.
\end{abstract}

